\title{
Investigation of activated carbon coating in the adsorption process of methylene blue from aqueous solution
}

\begin{abstract}
(GAC) on high-density polypropylene (HDPE) in the attempt to use coated HDPE for adsorption applications. Four HDPE substrates were coated by using either sol-gel method, namely TEOS, or Paint- Spray-Dry method, namely applying epoxy resin to HDPE using brushing technique and spraying the AC on the epoxy layer. The ability of these coated surfaces to remove methylene blue (MB) were then investigated. In addition, the durability of the coatings was characterized by using Energy Dispersive X-ray (EDX) and Scanning Electron Microscopy (SEM) on Day 1 and Day 28. Results show that the activated carbon demonstrated a good adsorption capacity his research evaluates various coating methods and conditions of granular activated carbon of $12.75 \mathrm{mg} / \mathrm{g}$ at $50 \mathrm{mg} / \mathrm{L}$ dye, indicating that regardless of whether the $\mathrm{AC}$ is coated or embedded in the epoxy layer, it could be considered as a promising material for the removal of MB dye from aqueous solution. The surface integrity analyses, such as shaking method, were conducted and their results confirmed that granular activated carbon coating has been successfully deposited on the HDPE substrate and that there was no loss of carbon between Day 1 to Day 28.
\end{abstract}

Keyword: Activated carbon; Methylene blue; Coating; Adsorption; HDPE 